\title{
La Descentralización de Servicios de Salud en Córdoba (Argentina): Entre la Confianza Democrática y el Desencanto Neoliberal (a)
}

\author{
Decentralization of Health Services in Córdoba \\ (Argentina): Among Democratic Confidence and \\ Neoliberal Disappointment
}

Iván Ase ${ }^{1}$

${ }^{1}$ Médico. Magíster en Administración Pública. Docente e investigador del Instituto de Investigación y Formación en Administración Pública de la Universidad Nacional de Córdoba y de la Universidad Nacional de Río Cuarto. Argentina. ivanase@ciudad.com.ar
RESUMEN La década del '90 ha sido rica en experiencias de reforma en el sector salud, procesos que deben ser ubicados en los cambios más generales que se han producido en las relaciones entre el Estado, la sociedad y el mercado. Así, el estudio de las políticas de descentralización de los servicios de salud en Argentina, y particularmente en Córdoba, adquiere importancia.

Este tipo de políticas fueron impulsadas debido a sus posibilidades en torno a mejorar la eficacia, eficiencia y calidad de los servicios, además de profundizar la democratización y participación comunitaria en la producción de los mismos. El desarrollo de dichas políticas en el contexto de los '90 impuso la lógica del ajuste fiscal en detrimento de la lógica sanitaria.

El trabajo destaca la importancia de los procesos políticos provinciales y locales a la hora de analizar los resultados obtenidos por las políticas de reforma de los servicios de salud.

PALABRAS CLAVE Reforma del Sector Salud; Reforma del Estado; Descentralización; Evaluación de Procesos y Resultados (Atención de Salud).

ABSTRACT The decade of the $90^{\prime}$ has been rich in experiences of reform in the health sector, processes that must be located in the most general changes that has been produced in the relations between the State, the society and the market. Therefore, the study of the policy of decentralization of the health's service in Argentina, and particularly in Córdoba adquires importance.

This kind of policies was propitiated due to its posibilities of enhance the efficacy, efficiency and quality of the services, furthermore inciting democratization and communitary participation in the production of these services. The development of these policies in the 90 's context submitted to the fiscal adjust logic in detriment of the sanitary logic.

The paper emphasizes the importance of subnational and local political processes in order to examine the outcomes of health services' reform policy.

KEY WORDS Health Sector Reform; State Reform; Decentralization; Outcome and Process Assessment (Health Care). 


\section{INTRODUCCIÓN}

La década del '90 ha sido rica en experiencias de reformas en el sector salud en Argentina. Este proceso ha estado enmarcado en los cambios más generales que se han producido en las relaciones entre los diversos niveles del Estado, la sociedad y el mercado.

En la provincia de Córdoba (b), a partir del año 1995, también se ha producido una importante transformación del sistema de salud provincial, que, con continuidades y diferencias, ha sido producto de las políticas de reforma sectorial Ilevadas adelante por los diferentes gobiernos provinciales desde entonces $(1,2)$.

Sin embargo, debe decirse que las políticas de descentralización no son un producto aparecido, ni en Argentina ni en la provincia de Córdoba, durante los años '90. En todo caso, durante la mencionada década adquirieron características que las alejaron de las ideas en el seno de las cuales surgieron.

Así, si las políticas de descentralización aparecen, desde mediados de la década del '50, al servicio de una ya necesaria racionalización de los sistemas de atención médica que permitiese alcanzar una mejor relación entre recursos invertidos y resultados sanitarios, durante la década del '90, por el contrario, la descentralización se puso al servicio, casi exclusivo, del "ajuste fiscal" y de una fuerte redistribución de responsabilidades en materia sanitaria entre las diferentes jurisdicciones estatales y, entre éstas, la sociedad y el mercado.

El fuerte sesgo "ajustista" que asumieron las políticas descentralizadoras y la subordinación de la racionalidad sanitaria a la racionalidad económica que esto produjo, tal vez sirvan para explicar, al menos en parte, los pobres resultados que las políticas de descentralización han producido, en particular, en América Latina. Al respecto, empieza a ser abundante la bibliografía que, a nivel regional, señala este problema (3).

Entonces, por lo dicho hasta aquí, es que consideramos de importancia realizar investigaciones de casos que aporten información sobre lo sucedido con el proceso de descentralización de servicios de salud en Argentina. En este sentido, el caso de la descentralización sanitaria en la provincia de Córdoba es paradigmático del recorrido antes señalado.

La descentralización de servicios sociales, y en particular los de salud, pasó a formar parte de la agenda de modernización del estado provincial de Córdoba a partir del año 1987, con la reforma de la Constitución Provincial. Sin embargo, y tal como ha sucedido en la mayoría de las experiencias descentralizadoras en América Latina, la descentralización de servicios de salud en Córdoba se concreta efectivamente a finales de 1995, apurada por una brutal crisis de financiamiento del estado provincial, lo que lleva al gobierno a tomar medidas de ajuste fiscal extremo con el objetivo de mejorar el estado de las cuentas públicas provinciales a muy corto plazo. En este contexto, se produce, en un lapso de seis meses, la transferencia masiva de efectores sanitarios de baja complejidad desde la jurisdicción provincial a la municipal.

Así, el presente trabajo intentará realizar una caracterización del proceso de descentralización de servicios de salud Ilevado adelante en la provincia de Córdoba, analizar el proceso de implementación de la política de descentralización y evaluar los resultados obtenidos en comparación con el diagnóstico realizado al inicio del proceso por las autoridades ministeriales del momento, lo que justificó la propuesta política de descentralización de servicios de baja complejidad.

A lo largo del trabajo argumentaremos a favor de cuatro hipótesis que han orientado el mismo. La primera de ellas sostiene que el proceso de descentralización de servicios de salud desarrollado en Córdoba no permitió obtener los efectos virtuosos esperados inicialmente.

Esto ha sido así ya que, como segunda hipótesis de trabajo, consideramos que el proceso de implementación de la política de descentralización estuvo guiado por una lógica economicista de corto plazo, que condicionó la modalidad, secuencia y los tiempos del proceso.

La tercera hipótesis, por su parte, afirma que la lógica predominante en el proceso de descentralización impidió que quienes impulsaban el proceso desde el nivel central provincial, favorecieran el desarrollo de capacidades institucionales o de gestión imprescindibles para optimizar los resultados del mismo. 
Finalmente, y como último supuesto de trabajo, se afirma que los resultados del proceso de descentralización, también, estuvieron determinados por la posición (compromiso, pasividad, resistencia) que asumió cada municipio en relación al proceso impulsado por el gobierno provincial.

El desarrollo del trabajo se estructurará en dos partes. En la primera se desarrollarán los rasgos que tuvo el proceso de descentralización de servicios de salud en Córdoba, señalando las diferentes etapas del mismo. En la segunda, se analizarán los resultados obtenidos en términos del funcionamiento del sistema de salud a nivel local. Al finalizar el informe, y en función de las conclusiones a las que arribamos, se dejan planteadas posibilidades de investigaciones futuras que nos permitirían avanzar en una mejor comprensión del proceso de construcción de la institucionalidad sanitaria a nivel local.

\section{LA DESCENTRALIZACIÓN DE SERVICIOS DE SALUD EN CÓRDOBA}

Las políticas de descentralización de servicios de salud han tenido un papel relevante en los procesos de reforma sanitaria, tanto en la Argentina como en toda América Latina. Sin embargo, como ya señalamos, estas políticas no constituyen un caso típico de política surgida en el marco de los procesos de reforma estatal de cuño neoliberal propios de los '90. Por el contrario, las propuestas descentralizadoras en el campo sanitario tienen antecedentes que se remontan a la década del '50 (4). Tal vez, lo novedoso ha sido el impulso que tomaron a la luz de los citados procesos.

Así, la descentralización, entendida como el proceso a través del cual la autoridad se sitúa lo más próximo a donde se suscitan los problemas o situaciones que ameritan algún tipo de decisión y que por ello implica la transferencia de responsabilidades, capacidades y recursos desde un nivel superior de gobierno a otro inferior (5), tiene una larga historia en nuestro país. Lo que sí ha variado a lo largo del tiempo, es el énfasis con que se ha avanzado sobre esta política.

También, a lo largo de estos años, se fue complejizando el conjunto de motivaciones que servían como justificativos del proceso descentralizador. En este sentido, la descentralización se fue transformando en una política que en diferentes planos abordaba diversas problemáticas.

Pues bien, la descentralización apuntaba al logro de tres grupos de objetivos: a) mejorar la eficacia y calidad de los servicios de salud; b) aumentar la eficiencia en la utilización de los recursos; y c) democratizar los servicios de salud. Esto convirtió al proceso descentralizador en "el remedio" para los múltiples males que aquejaban, y aún hoy aquejan, a los sistemas sanitarios.

No obstante lo anterior, es abundante la bibliografía nacional e internacional que señala que, durante los procesos de implementación de estas políticas en las últimas décadas, y en especial durante los '90, una "lógica economicista" (6) ha conducido el proceso. Las urgencias fiscales y un clima intelectual de rechazo a "lo estatal" impulsaron procesos de transferencia de servicios sanitarios y sociales hacia los niveles locales, que tenían como único objetivo mejorar, en el corto plazo, la "posición" fiscal del nivel central en relación a las jurisdicciones subnacionales pero sin tener en cuenta las capacidades locales disponibles para hacerse cargo de las nuevas tareas.

En este contexto, la descentralización de servicios de salud implicó, en la mayoría de los casos, una fuerte pérdida en la calidad de los servicios, y ha acentuado la heterogeneidad en las respuestas sanitarias que cada jurisdicción elabora, profundizando las desigualdades regionales y provocando enormes costos en términos de eficiencia y equidad.

En el caso de la provincia de Córdoba, las políticas de descentralización han formado parte de los discursos sanitarios oficiales desde hace por lo menos treinta años. Pero nuestro interés se centra en el proceso de descentralización de servicios sanitarios que se desencadena a partir de la reforma de la Constitución Provincial de 1987, en la medida en que, a partir de ese momento, la descentralización de los servicios de salud adquiere un "paraguas" normativo de tipo constitucional.

En este sentido, debe decirse que el proceso de descentralización estuvo marcado por características singulares en función de las variaciones que iba sufriendo el contexto político y 
económico provincial. Por ello, es posible observar que el proceso tiene etapas claramente diferenciadas: una, de tipo "gradualista" (período 1988 - julio 1995), en donde lo que primó fue la búsqueda de consenso entre los actores involucrados; y otra, de shock (julio 1995 - 1999), en la que primó la urgencia y compulsividad en la transferencia de los efectores de salud. Además, existiría un tercera etapa (1999 - 2005) durante la cual, a pesar de la inexistencia de efectores a transferir, el objetivo sería consolidar lo hecho mediante la coordinación e integración de los efectores "municipalizados" en el marco de un nuevo "modelo" de salud provincial.

El proceso de descentralización en Córdoba, entonces, adquiere una doble relevancia. Por un lado, el alto nivel de consenso entre los actores políticos provinciales, que ha provocado que gestiones de distinto signo político hayan decidido sostenerla en el tiempo, convirtiéndola, más allá de los matices, en una verdadera "política de estado". Por el otro, la profundidad que ha adquirido el proceso descentralizador a lo largo de los años ha hecho que la provincia de Córdoba tenga un altísimo nivel de involucramiento municipal en la producción de servicios de salud en comparación con otras provincias argentinas.

\subsection{ETAPA "GRADUALISTA"}

La descentralización de servicios sociales, y en particular los de salud, pasó a formar parte de la agenda reformista del gobierno provincial de Córdoba a partir del año 1987, con la reforma de la Constitución Provincial. Esta política se materializa inicialmente a través del marco jurídico elaborado por el gobierno provincial de entonces. De dicho marco formaban parte el decreto 1174/88 (Políticas generales y Pautas normativas para los programas de descentralización de la Provincia) y la ley de Reforma Administrativa 7850/89.

En este marco legal, la provincia avanza en la definición de la política de descentralización y elabora un crudo diagnóstico de la situación sanitaria provincial al momento de iniciarse este proceso. Los problemas generados por una dinámica excesivamente centralizada de funcionamiento del estado provincial ocupan un lugar central en el mismo.

Las causas de las ineficiencias del sector sanitario estatal se podían resumir en el listado que sigue:

a. Deficiente asignación de recursos humanos y materiales.

b. Desinformación y escasa participación de autoridades, funcionarios y organizaciones comunitarias a nivel local.

c. Imperfecciones del sistema de fiscalización y control.

d. Inadecuado sistema de compras, adjudicación y distribución de insumos.

e. Inadecuados, y a veces inexistentes, estudios para la determinación de la demanda de atención, su estructura y dinámica.

f. Escaso compromiso con la unidad de servicio y la comunidad, y cumplimiento inadecuado con la actividad funcional por parte de los profesionales debido a sus bajos ingresos relativos, su dedicación parcial, su falta de conocimientos epidemiológicos del área de trabajo y la falta de un sistema eficiente de control administrativo.

g. Inadecuado sistema de programación y presupuestación a nivel general, con mayores dificultades a menores niveles de complejidad.

h. Insuficiente, y a veces inexistente, sistema de informática y escaso o nulo equipamiento en tecnologías administrativas, acentuado en los menores niveles de complejidad.

i. Escasa o nula coordinación intersectorial, interjurisdiccional o entre los subsectores prestadores.

j. Imperfecciones en los esquemas y procedimientos de control de gestión en todas las áreas del Ministerio de Salud.

k. Imperfecciones en el funcionamiento del esquema zonal, en donde la zona sanitaria es un mero ente administrativo con escasa, y a veces nula, participación en la gestión del sistema de salud a nivel regional (7).

En este contexto de dificultades, la normativa provincial define el tipo de efectores a considerarse como "descentralizables". Así, se establece que la provincia avanzaría en el traspaso a nivel municipal de todos los efectores calificados como 
de baja complejidad, guardándose para sí los de complejidad media y alta. Esto sería así ya que se considera que las actividades de los efectores de baja complejidad involucran casi con exclusividad al nivel local, mientras que las de los efectores de mediana y alta complejidad involucran a una magnitud poblacional que trasciende a la de un municipio o comuna. Al iniciarse el proceso de descentralización, de los 467 establecimientos provinciales, 418 eran considerados como "descentralizables". Debe decirse que, hasta la década del '90, la prestación del servicio público de salud en la Provincia de Córdoba se encontraba casi exclusivamente bajo responsabilidad provincial, y sólo los municipios más grandes (Córdoba y Río Cuarto, en particular) contaban con servicios de salud municipales que coexistían con los provinciales.

La modalidad adoptada para la implementación de la transferencia de efectores a los municipios consistía en la adhesión a un convenio marco entre el nivel central y el local en donde se definían las obligaciones y derechos de cada una de las partes. La firma de los convenios era el producto de trabajosos procesos de negociación a través de los cuales se buscaba alcanzar altos niveles de consenso entre los actores involucrados.

Principalmente, en estos convenios se establecía que la provincia cedía al municipio o comuna los bienes muebles e inmuebles afectados al efector descentralizado, pero el recurso humano seguía perteneciendo a la jurisdicción provincial. El gobierno local se comprometía a garantizar el funcionamiento y mantenimiento edilicio del efector sanitario $y$, en la medida en que se produjeran vacantes, iría ocupándolas con personal municipal. Para cumplir con estos objetivos, el financiamiento se garantizaría mediante la afectación del $12,5 \%$ del monto global de coparticipación, con el que se conformaría el Fondo de Financiamiento de la Descentralización (FOFINDES).

Ahora bien, el proceso de descentralización no sólo avanzó lentamente bajo estas condiciones, sino que también tuvo importantes dificultades. Evaluaciones realizadas sobre la marcha determinaban que muchos de los convenios efectivamente firmados sufrían innumerables complicaciones de implementación que los ponían al borde de ser "denunciados". Entre las dificultades salientes se pueden detallar: imposibilidad económica de los municipios para designar personal propio, falta de determinación de costos de los servicios transferidos, insuficiencia de fondos transferidos, incumplimiento por parte de las autoridades de Zona Sanitaria de las funciones asignadas, incomprensión por parte de las autoridades locales del perfil que debe tener un efector local de baja complejidad (muchos intendentes decidieron invertir recursos en aparatología o en habilitar salas de internación en los efectores recibidos), ineficiencias en el uso de recursos financieros al perder "escala" en la adquisición de insumos y la doble dependencia del recurso humano (8).

No obstante lo anterior, el gobierno central logró que en esta primera etapa del proceso de descentralización se lograran acuerdos que involucraban al $24 \%$ de efectores "descentralizables". Así, según información oficial, en el año 1994 se encontraban en vigencia 117 convenios de descentralización en el ámbito del sector provincial de la salud (9).

El "gradualismo" en el proceso de implementación no puede ser analizado sólo desde las necesidades técnicas del mismo; por el contrario, es también la incorporación de los factores políticos lo que nos permitirá tener una comprensión más acabada de lo sucedido con la política descentralizadora en este período.

En este sentido, el proceso de descentralización, formaba parte durante este período de un proyecto mayor de reforma del Estado que intentaba diferenciarse claramente del que se Ilevaba adelante desde el gobierno nacional. Este "modelo cordobés" de reforma estatal apuntaba a mejorar la gestión del Estado pero sin afectar de manera profunda las bases del sistema "bienestarista" provincial (10). Para ello, el gobierno provincial logró eludir las restricciones fiscales que ya padecían en ese momento otras jurisdicciones del país y "manejar" los tiempos políticos de los procesos de transformación estatal iniciados. Los flujos financieros provenientes desde el exterior a partir del Plan de Convertibilidad y el rol de la banca provincial con relación a los mismos posibilitaron este comportamiento.

Así, el ritmo y la característica "acuerdista" que tuvo esta primera etapa descentralizadora 
habrían estado determinadas por las necesidades políticas de quien ejercía dicho liderazgo en la provincia. La transferencia de recursos desde el nivel central hacia los municipios sin la clara contraparte de los mismos, en el sentido de asumir nuevas responsabilidades sanitarias o sin los controles necesarios para determinar el destino de esos fondos a nivel local, formó parte de los recursos de poder necesarios para estabilizar el complejo entramado de poder territorial. Las necesidades con relación a consolidar un liderazgo hegemónico, de tipo "conservador", tanto hacia adentro como hacia afuera del propio partido (Unión Cívica Radical), habrían sido las que marcaron los tiempos y modos de la descentralización de servicios de salud en este período.

Finalmente, debe decirse que a la hora de evaluar lo acontecido en esta etapa del proceso descentralizador, en el trabajo de investigación surgen dos perspectivas de interpretación de lo sucedido. Por un lado, quienes acuerdan con los rasgos que adquirió el proceso resaltan su estricta "lógica sanitaria" y las ventajas "de largo plazo" que generaría el mismo. Por el contrario, quienes lo cuestionan por su "lentitud" y sus dificultades para transformar la estructura sanitaria provincial dentro de un plazo razonable, sostienen que esto fue producto de que el proceso de descentralización estuvo subordinado a una estrategia global de construcción de poder provincial.

Seguramente, ambas interpretaciones no son excluyentes entre sí y no hacen más que mostrar la complejidad de un proceso caracterizado por la presencia de actores portadores de ideas, intereses, proyectos y estrategias diversas.

\subsection{ETAPA DE "SHOCK"}

A finales de 1994 e inicios de 1995, la crisis financiera internacional desatada por la devaluación mejicana ("efecto tequila") terminó con el conjunto de condiciones político-económicas que el gobierno provincial había usufructuado y le habían permitido postergar los inevitables "ajustes" del modelo "bienestarista" provincial. Así, la ilusión de una "isla cordobesa" terminó estallando en un mar de escasez de recursos, lo que finalmente evidenció la necesidad de impulsar un programa de ajuste y reforma del estado provincial que le devolviera a éste su solvencia financiera.

En dicho contexto económico, y en el marco de amplias movilizaciones sociales provocadas por la crisis, se produce el primer recambio de gobierno provincial desde el retorno a la democracia en 1983. El nuevo gobierno, a pesar de ser del mismo signo partidario que el anterior, considera la crisis como un punto de inflexión en la política provincial, lo que lo habilitaría para llevar adelante un ajuste de magnitud inusual e iniciar reformas de sectores considerados clave en una nueva arquitectura estatal.

Así, la crisis fiscal y financiera fue utilizada como fuente de derecho a los fines de implementar medidas que restituirían el orden político y financiero, pero también para avanzar sobre reformas de sectores (salud y educación, por ejemplo) que se consideraban incapaces de dar respuestas a los nuevos desafíos de la época. Todo este proceso fue altamente conflictivo y costoso en términos de capital político por lo que implicaba en términos de redistribución social de los costos de la crisis del estado y del desmejoramiento de la situación relativa de amplios sectores.

En cuanto al proceso de descentralización, el gobierno critica tanto la lentitud con que se venía implementando como el hecho de que los municipios se estaban beneficiando con fondos específicos (FOFINDES) sin asumir las responsabilidades sanitarias que les correspondían. De esta forma, la nueva administración se propone profundizar y consumar el proceso tibiamente desarrollado hasta ese momento, reconociendo expresamente los objetivos fiscales de la descentralización. Bajo una modalidad de descentralización masiva (a todos los municipios y comunas), acelerada (en alrededor de seis meses) y forzosa (presión financiera y amenaza de cierre de servicios), se transfiere el efector y su personal con carácter definitivo (11). En 1996, la provincia había transferido la totalidad de los efectores "descentralizables".

Mediante negociaciones que fueron conducidas personalmente por el gobernador, $y$ en las cuales se discutía con los intendentes no sólo sus nuevas responsabilidades sanitarias, sino también, y sobre todo, los montos de coparticipación que serían transferidos hacia el futuro, los gobiernos locales, con escasas excepciones, 
terminaron firmando los convenios de transferencia impulsados por el nivel central. Finalmente, la provincia municipaliza 526 efectores en 6 meses, en especial aquellos correspondientes al primer nivel de atención, el cual, según el gobierno, debía pasar a ser responsabilidad exclusiva de los gobiernos locales. La provincia se comprometía a garantizar el segundo y tercer nivel de complejidad.

La oposición al proceso estuvo centrada particularmente en el estilo de gestión del gobierno, que evitó sistemáticamente la creación de espacios de discusión y búsqueda de consensos que sirvieran para viabilizar sus políticas. Este estilo, además de relacionarse a las características personales del gobernador, reflejaba una singular concepción de la representación política, en sintonía con la modalidad emergente en la mayoría de los procesos de ajuste y reforma en el país y la región. El mismo se caracterizó por ser marcadamente personalista, conformando una suerte de "decisionismo" basado en la legitimidad electoral que emanaba de la figura del primer mandatario provincial, lo que lo impulsaba a llevar adelante sus políticas para el sector sin necesidad de participar a los actores involucrados.

Así, durante este período fue notoria la ausencia de instancias mediadoras a las cuales se convocara a los actores, neocorporativos y/o sociales, con el fin de construir espacios de entendimiento en torno a las políticas a implementar.

A decir verdad, la reforma en su conjunto era presentada por el gobierno como un proceso de disciplinamiento de los actores sectoriales de la salud, los cuales se habían sobreexpandido en otras épocas, en torno al interés superior del "bien público" que el gobierno representaba.

En definitiva, todo este proceso de reforma del sector salud estuvo conducido por un estilo personalista, plebiscitario y con escasa participación de los actores involucrados, a quienes, a su vez, no se consideraba con derecho a hacerlo. Este proceso pretendía ser evaluado por sus resultados y no por los medios con que se obtenían.

De esta manera, la falta de instancias mediadoras, sumada a las restricciones financieras del gobierno que le impidieron crear incentivos "pro-reforma", produjo un escenario de alta conflictividad con escasas posibilidades de lograr consenso en torno a ideas.
Finalmente, debe decirse que el proceso de descentralización impulsado al ritmo de las urgencias fiscales y en el marco de un clima francamente hostil entre los actores involucrados, no le permitió al gobierno generar los mecanismos mínimos de cooperación entre los actores. Esto hubiera permitido construir la red de efectores de complejidad creciente que, según el proyecto gubernamental, debía rodear como "una malla de contención" a la capital provincial. Tampoco pudo avanzar sobre la instrumentación de políticas que permitieran fomentar el desarrollo de capacidades de gestión en los municipios que se hacían cargo, de forma novedosa para ellos, de la prestación de un servicio de suma complejidad. Por el contrario, la suerte de los efectores descentralizados quedó librada a las estrategias que cada localidad, sola o articulada con otras cuya vinculación se daba por cercanía geográfica, podía impulsar espontáneamente a los fines de sostener los servicios sanitarios locales.

\subsection{ETAPA DE "CONSOLIDACIÓN"}

La gestión del actual gobernador provincial, José Manuel de la Sota, se inicia en julio de 1999 con un fuerte compromiso de revertir el conjunto de las políticas de reforma sectorial impulsadas por la gestión anterior. Este compromiso no sólo formó parte de sus promesas electorales, sino que también fue el eje de los compromisos asumidos con algunos de los actores sectoriales más significativos. Esto cristalizó en la designación como ministro de Salud de la gestión que se iniciaba al entonces presidente del Consejo de Médicos de Córdoba y a varios dirigentes gremiales hospitalarios en cargos de relevancia.

Sin embargo, con relación al tema de la descentralización, el gobierno decidió considerarlo como un "hecho consumado" de imposible reversión. Esto fue así debido a dos razones. Por un lado, el gobierno cuestionó el "modo" de implementación pero refiere acordar con la nueva distribución de responsabilidades sanitarias entre las diferentes jurisdicciones de la provincia. Por el otro, a pesar del fuerte rechazo generado inicialmente, los intendentes y jefes comunales en ningún momento plantearon seriamente la "devolución" de los servicios a la provincia. 
En todo caso, lo que el nuevo gobierno sí decidió, fue recuperar el rol de la jurisdicción central en la articulación y coordinación de los efectores municipales. Para transformar el tipo de relación con los municipios y comunas, el gobierno, que convalida el proceso realizado, saca provecho de una situación que le permite descargar los "costos" del mismo sobre la gestión anterior y presentarse como quien padece los límites y restricciones propias de una acción "reparadora" de los "daños" producidos por otro gobierno.

En su relación con los municipios es posible determinar dos etapas, coincidentes con las dos gestiones ministeriales que han conducido el área salud desde entonces. En una primera etapa (1999 - 2001), se le da a la problemática de la descentralización un carácter eminentemente político. Esto queda evidenciado en que la relación con los municipios se conduce desde el Ministerio de Gobierno Provincial, el cual realiza un diagnóstico lapidario sobre la situación del sector salud en el interior de la provincia, al que define en estado de anarquía. El citado diagnóstico se asemeja de manera llamativa al realizado por el estado provincial al inicio del proceso descentralizador.

Debe decirse aquí que la participación en este tema de un ministerio eminentemente político y carente, seguramente, de los recursos técnico-sanitarios necesarios sólo se explica por el aprendizaje que este gobierno hizo sobre la gestión anterior. Es decir, haber entendido la enorme trascendencia política que tenía la descentralización de servicios de salud en orden a construir, en una primera etapa de la gestión, una estructura de poder estable y confiable para el gobierno en el interior de la provincia.

En este contexto, el Ministerio de Gobierno lanza en el año 2000 el "Plan de Salud Municipal" en el marco de la recién creada Mesa Provincia-Municipios, instancia ideada para darle un cauce institucional a la relación política entre el Gobierno Provincial y los diferentes gobiernos municipales y comunales. En este ámbito, luego de meses de discusión, se logra plasmar una acuerdo con alto nivel de consenso entre los involucrados, con relación a los modos de distribución de los recursos destinados a los efectores descentralizados y a la manera en que el Estado
Provincial asumiría un rol más activo en el proceso descentralizador, en cuanto se lo considera co-responsable de los servicios de salud que brindan los municipios y comunas. El acuerdo queda plasmado en el Decreto Provincial № 2640 del 15 de Diciembre de 2000.

A partir del segundo semestre del 2001, el Gobierno Provincial produce un recambio de algunos de sus ministros y se replantean las estrategias políticas para algunas de sus áreas más importantes. Un gobierno más consolidado en su gestión decide iniciar una segunda etapa (2001 2005) en su política de salud mediante la designación de "un técnico del partido de gobierno" como ministro del área, el cual, a su vez, había sido uno de los responsables del acuerdo con los municipios. Desde ese momento, la política sanitaria se centraliza en torno al ministerio específico y, en relación con la política de descentralización, se impulsan las políticas acordadas en el marco del Decreto Provincial No 2640/00.

Ahora bien, llegados a este punto es necesario reseñar cuáles han sido las políticas diseñadas para avanzar en el proceso de consolidación de la descentralización.

En primer lugar, las autoridades ministeriales avanzan en el proceso de regionalización con el fin de crear estructuras intermedias que faciliten las relaciones intermunicipales $y$ entre los municipios y el nivel central. Así, se crean 9 regiones sanitarias no en función de las divisiones departamentales, sino siguiendo afinidades o criterios de vinculación diferentes (culturales, geográficos, de accesibilidad, entre las más importantes) a los fines de garantizar un funcionamiento más aceitado de las mismas. Debe recordarse que la administración anterior, como parte de la política de achicamiento de las estructuras político-administrativas del Ministerio de Salud, había desactivado las Ilamadas Zonas Sanitarias que tenían funciones de coordinación regional.

En segundo lugar, el Ministerio, en su relación con los municipios, ha reforzado su rol financiador en detrimento de su rol de prestador. En este sentido, frente a diferentes reclamos vinculados a la provisión de insumos o de algún tipo de tecnología, el Ministerio ofrece los recursos financieros necesarios para cubrir la necesidad, pero a cambio de reforzar el rol del municipio en 
cuanto a la prestación del servicio. Esto es así ya que las autoridades esperan que el municipio logre resolver la mayor cantidad de problemas en el lugar en el que se producen, y evitar así la llegada de pacientes a la capital provincial.

En tercer lugar, en cuanto al tema del financiamiento, el Ministerio está intentando impulsar algunas innovaciones en relación con los criterios de asignación del FOFINDES. En este sentido, aquellos municipios que derivan permanentemente pacientes descompensados a otras localidades por falta de atención o controles en su propia localidad, el Ministerio los "castigaría" reduciendo el aporte correspondiente del fondo, monto que, a su vez, se transferiría al municipio cuyo efector ha recibido al o los pacientes. Esta estructura de premios y castigos en la asignación de los recursos busca comprometer a los municipios y responsabilizarlos por los resultados sanitarios que se obtienen en su área jurisdiccional.

En cuarto lugar, con el objetivo de fortalecer la atención local, el Ministerio está implementando el Programa de Salud Familiar que se considera como una novedosa estrategia descentralizada de atención a zonas de mayor riesgo y vulnerabilidad sanitaria. Dicho programa tiene por objetivo fortalecer el primer nivel de atención con recursos provinciales, también con el fin de evitar la llegada de pacientes a los hospitales de Córdoba capital. Para esto se han distribuido equipos interdisciplinarios de salud familiar a lo largo de toda la provincia, y cuyo trabajo pone énfasis en la prevención y promoción de la salud. Esta estrategia implica un importante trabajo de coordinación con las autoridades locales a los fines de evitar superposiciones y lograr complementariedad en las acciones. La intención del gobierno sería lograr la coordinación e integración de todos los efectores provinciales, públicos o privados, en redes de complejidad creciente y en donde el primer nivel de atención ("la puerta de entrada al sistema") tendría una importancia sustancial.

\section{EL SISTEMA LOCAL DE SALUD}

La descentralización de servicios de salud, como ya se dijo, se suponía que sería capaz de lograr resultados satisfactorios en términos de eficiencia, eficacia, calidad y participación. No sólo esto: se creía también que sería la estrategia adecuada para avanzar en la transformación del modelo médico prestacional, excesivamente asistencialista y centrado en la enfermedad.

En definitiva, el planteo teórico en relación con la descentralización suponía que la política de salud "decidida" a nivel local haría más eficiente la asignación de los recursos, mejoraría los indicadores sanitarios, sería más receptiva a las preferencias y demandas ciudadanas y, sobre todo, ayudaría a democratizar la gestión del estado.

En este sentido, al intentar precisar si el proceso descentralizador fue capaz de acercarse a los objetivos planteados inicialmente, se debe decir que los resultados del mismo no sólo han estado influenciados por la "lógica" con que se lo condujo desde el nivel central, sino también por la actitud asumida por los gobiernos locales en relación con el mismo.

Pues bien, la mayoría de los intendentes, a pesar de ciertas dudas e incertidumbre iniciales en cuanto a lo que significaba administrar una institución de salud, coincidían con la "idea" descentralizadora. Lo que reclamaban era "tiempo" para poder adaptarse a la nueva situación y garantías en cuanto a la transferencia de los recursos económicos necesarios para financiar el funcionamiento de los centros sanitarios. En sí, no es posible encontrar entre los actores políticos principales del sector a nivel local una oposición de tipo conceptual al proceso descentralizador, sino que, por el contrario, la mayoría de las críticas y reparos están centradas en las "formas" en cómo se terminó Ilevando adelante el proceso. En particular, al excesivo "economicismo" que tiñó a la segunda etapa del mismo.

Ahora bien, más allá del relativo consenso general que tenía y tiene aún hoy el proceso de descentralización de servicios de atención médica, la forma que adquirió su implementación generó diferentes comportamientos entre los municipios a la hora de "recibir" y gestionar las instituciones sanitarias.

Estas diferencias no pueden ser atribuidas a causas únicas y, mucho menos, universales. Por el contrario, las particularidades políticas, sociales, económicas, históricas, culturales y hasta personales de cada intendente son las que permitirían comprender, en cada caso en 
particular, la institucionalidad sanitaria construida a nivel local.

Sin embargo, con el afán de producir agrupamientos que nos permitan identificar los diversos comportamientos adoptados a lo largo del proceso, es posible afirmar que se han podido registrar tres tipos de conductas con relación a la descentralización:

- Aceptación activa: se trata de aquellos municipios que aceptaron la transferencia de servicios y que, además, han gestionado los mismos con un criterio expansivo. Es decir, han tomado el desafío de llevar adelante una política de salud que tenga como resultado un servicio que mejore en cantidad y calidad lo recibido.

- Aceptación pasiva: se trata de aquellos municipios que no se resistieron al traspaso de efectores y los recibieron con resignación al evaluar como poco probable la posibilidad de impulsar una acción que tuviera chances de cambiar la decisión del gobierno provincial. Sin embargo, lo que hicieron de forma más o menos inmediata fue "achicar" el efector recibido, en algunos casos al punto, de plantear el cierre liso y llano del mismo.

- Resistencia: se trata de aquellos escasísimos casos en los cuales hubo un proceso de confrontación abierta con el gobierno provincial en cuanto a la aceptación de las condiciones de transferencia de los efectores. Debe decirse que esta resistencia, en todos los casos, sólo permitió demorar el traspaso del efector asignado, cosa que finalmente se concretaría, aunque con un convenio "mejorado" para el municipio en comparación al originalmente propuesto.

Debe decirse que estas diferentes posiciones con relación a la descentralización aún hoy generan controversias y discusiones a nivel local que, en muchos casos, entorpecen la posibilidad de trabajar articuladamente entre los municipios. En particular, se critica la actitud de aquellos municipios que decidieron "desactivar" sus instituciones de salud ya que, se considera, esto es posible porque le "descargan" la problemática de la salud a los municipios vecinos o a los efectores provinciales.
En realidad, lo arriba expuesto pone de manifiesto una situación crítica y que confronta a la jurisdicción provincial con sus más que evidentes límites en términos de capacidad de coordinación de la acción de los municipios y comunas en torno a la cuestión salud.

A continuación se señalarán algunos de los rasgos sobresalientes que caracterizan al funcionamiento del sistema de salud a nivel local y regional luego del proceso descentralizador. Los mismos deberán ser contrastados con las características que poseía el sistema sanitario al iniciarse el proceso de descentralización, y así se podrán precisar los resultados obtenidos y las brechas entre los objetivos propuestos y los logros efectivamente alcanzados.

\subsection{GRADO DE COORDINACIÓN}

La descentralización implementada en el marco de las urgencias financieras provinciales ha producido un verdadero "estallido" del sistema público de salud. La misma ha provocado una profundización de la ya existente atomización y fragmentación en el sistema, que obstaculiza la posibilidad de "construir" una respuesta racional e integrada a la problemática sanitaria provincial. Las capacidades estatales provinciales de coordinación tienen serias dificultades para producir articulaciones interjurisdiccionales, respuestas escalonadas y progresivas, y una "división del trabajo" y de las responsabilidades según las complejidades acordadas y que sea consensuada por todos los actores.

Debe recordarse aquí que la coordinación estatal, tarea de por sí compleja, adquiere en la provincia de Córdoba una dimensión ciclópea debido a las características de su organización político-institucional. En este sentido, la normativa institucional provincial ha provocado la creación, en los últimos 20 años, de una enorme cantidad de municipios y comunas que dificultan cualquier intento de coordinación desde el estado provincial. Así, en la actualidad, con una población de 3.066.801 habitantes (12), la provincia de Córdoba está dividida en 26 Departamentos y éstos, a su vez, se subdividen en 249 municipios y 178 comunas. 
En este marco, luego de 10 años de finalizado el proceso de municipalización de efectores sanitarios, no ha sido posible aún, a nivel provincial ni regional, consensuar un acuerdo que establezca para cada uno de los actores los márgenes dentro de los cuales debe prestarse el servicio de salud a su cargo. Esto hace que las características que asume cada efector de salud municipal dependan exclusivamente de la decisión política del intendente al respecto. Así, la decisión de "achicar" servicios o aumentar los mismos es una cuestión sobre la cual la jurisdicción de la provincia, tiene escasa capacidad de influencia. Incluso la idea de que el municipio debe limitarse a "la atención primaria" y al primer nivel de atención, idea que tiene un importante nivel de consenso, es pasible de múltiples interpretaciones sobre lo que esto significa en cuanto a tipo de servicio y cantidad de especialidades.

El nivel provincial se destaca, en la relación con los municipios, por la importante capacidad de la que dispone para resolver los problemas de cada gestión local. Sin embargo, se muestra débil en sus posibilidades de utilizar estas potencialidades como herramienta de coordinación entre los actores locales.

\subsection{VALORES Y PRINCIPIOS}

La atomización y fragmentación en el sistema de servicios de atención médica municipales, así como las dificultades de coordinación provinciales, no son los únicos rasgos del sistema emergente. También existe un fuerte proceso de dispersión en el terreno de los valores y principios sobre los que funciona el sistema de salud municipal, lo que ha provocado, a su vez, un importante incremento de la heterogeneidad dentro de la fragmentación (13).

Así, los valores y principios sobre los que se construyó el sistema público de salud en la Argentina de la posguerra, tales como derecho a la salud, universalidad, gratuidad e integralidad adquieren a nivel local significados y sentidos diferentes.

La oferta de servicios de salud locales está atravesada por un "mosaico" de sentidos que va conformando entramados institucionales absolutamente diferentes en el terreno sanitario. Este fenómeno no respeta las identidades tradicionales, tanto ideológicas como partidarias. Es la figura del intendente con su historia, sus valores, sus principios, sus intereses, en el marco de un particular entramado institucional, la que perfila la oferta de salud local. La política de salud, así, adquiere un nivel de discrecionalidad Ilamativo.

Ejemplo de lo dicho en el párrafo anterior es lo que sucede con los principios de universalidad y gratuidad. Así, a nivel local, es posible decir que, más allá de algunas excepciones, el consenso en torno a la universalidad está seriamente resquebrajado. En la mayoría de los casos se plantea explícitamente la necesidad de instrumentar restricciones, monetarias o de otro tipo, a los fines de que los servicios atiendan exclusivamente a los sectores carenciados, justificando esta posición debido a la escasez de recursos o, en otros casos, a cuestiones morales. También la focalización aparece motivada en el hecho de que el efector comunal no se convierta en una competencia para el sector privado de la salud local.

El tema de la necesidad de focalizar los servicios de salud está vinculado con el segundo tema, que es la gratuidad. En la mayoría de los actores aparece como predominante el consenso en torno a la postura que tienen los municipios de buscar formas alternativas de financiamiento. En este sentido, aparece la idea de responsabilizar a la sociedad a la que sirven a través del financiamiento compartido de los servicios y de las instituciones. Los modos de implementar esta idea son diversos, pero sobre lo que no hay dudas es acerca de la decisión de que la gratuidad absoluta se sostiene sólo para los carenciados de la propia comunidad.

\subsection{MODELO PRESTACIONAL}

Pero así como decimos que la descentralización ha provocado una profundización de la atomización y fragmentación del sistema de salud provincial, tanto en sus aspectos institucionales como en los principios que lo sustentan, es cierto también que ha sido útil para consolidar algunos rasgos del tradicional sistema de salud nacional y provincial, esto es, el modelo prestacional. Se suponía que la transferencia de efectores al nivel 
local facilitaría la transformación de los servicios en el sentido de abandonar los modelos puramente asistencialistas a favor de modelos de trabajo basados en programas, con un fuerte énfasis en lo preventivo y la promoción de la salud, y con un importante trabajo comunitario extramuros con equipos interdisciplinarios.

Por el contrario, los servicios descentralizados han consolidado modelos de trabajo centrados en la demanda espontánea, con la figura del médico como eje del servicio y escaso trabajo comunitario. La promoción de la salud o la prevención se limita a esporádicas acciones en escuelas, a la difusión de información a través de los medios de comunicación locales, al trabajo con clubes o instituciones barriales sobre temas de interés comunitario o a la tarea que se realiza en la propia consulta médica.

Además, la manera de legitimar el servicio que se presta a la comunidad es mediante la incorporación de "especialistas" de diverso tipo y de tecnología nueva o de mayor complejidad que la existente. Aunque por un lado se acepta que los municipios deben centrarse en consolidar el primer nivel de atención con las especialidades básicas, por el otro las autoridades municipales parecen considerar que "limitarse" a esto no satisface las expectativas de la comunidad en relación a "su" servicio de salud.

En este sentido, es más que evidente, una vez más, que el criticado Modelo Médico Hegemónico es un modelo que no sólo impregna las prácticas en salud actuales, sino que también se ha transformado en el "sentido común" comunitario en relación con lo que se considera "una buena medicina". Tecnologías de última generación y especialistas en los más variados campos parecen ser los componentes que transforman un servicio de salud local en "confiable" y "valorado" por su comunidad.

\subsection{REFERENCIA Y CONTRARREFERENCIA}

A todo lo anterior debe agregarse que, además de lo enumerado hasta aquí como rasgos del nuevo sistema descentralizado, otra cuestión que debe considerarse al caracterizar la manera en que están desarrollando sus tareas los servicios transferidos es la del relativo aislamiento en que trabajan los municipios y la falta de coordinación existente en la red regional de referencia y contrarreferencia.

A pesar de que a nivel central provincial está establecido el circuito de referencia y contrarreferencia que debe seguir cada paciente en cada lugar de la provincia, esto no logra plasmarse en un funcionamiento consensuado y ágil. Por el contrario, la falta de criterios establecidos o el no reconocimiento de los mismos hace que la red de derivaciones se ponga en marcha en base al amiguismo, las relaciones políticas o al voluntarismo de los actores intervinientes en cada ocasión. Aquí aparecen como déficit, nuevamente, las dificultades de coordinación que presenta la jurisdicción provincial.

Los municipios han intentado instrumentar las derivaciones "gestionándolas" de manera directa con los hospitales referenciales, para lo cual la mayoría de los mismos ha tercerizado el sistema de traslados de pacientes en ambulancias.

Otra de las razones que se evidencian para explicar las dificultades en el funcionamiento de la red de referencia y contrarreferencia es la sobrecarga de demanda que reciben los hospitales regionales de mayor complejidad. Esto sería así porque la presencia de estos grandes hospitales en ciudades del interior provincial es un incentivo a favor de que dichos municipios descarguen la atención de la salud de su comunidad en las citadas instituciones. De esta forma, los hospitales regionales terminan funcionando como los hospitales "de" la ciudad donde asientan, y sólo marginalmente ofrecen servicios a las comunidades de su área de referencia.

Una tercera razón que aparece a la hora de explicar las dificultades para funcionar en red es la profunda "subestimación" que dicen sufrir los médicos de las ciudades y comunidades pequeñas por parte de los profesionales de los grandes hospitales.

En este punto aparece nuevamente la vigencia del Modelo Médico Hegemónico, que sobrevalora al médico especialista de hospital en desmedro del médico clínico o generalista del dispensario o centro de salud local. En este marco cultural también prevalece la práctica individualista en detrimento del trabajo en equipo o en red, y que rechaza la posibilidad de aceptar la 
normatización o protocolización del trabajo profesional. La cultura médica "liberal" ha llevado la autonomía médica al rango de principio indiscutible de la ética profesional.

Finalmente, antes de concluir este apartado, debe hacerse mención a la manera en que los diversos modos de financiamiento de las instituciones de salud, implementados a nivel local, han agravado los crónicos problemas de los sistemas de derivación y de referencia y contrarreferencia.

En este punto, debe decirse que la aparición a nivel regional de la lógica del Hospital de Autogestión, de manera aislada y sin coordinación con el resto de los actores locales, aparece como un escollo en muchas ocasiones insalvables.

Los sistemas de referencia y contrarreferencia implican la construcción de una red de complejidad creciente de efectores, cuyo funcionamiento se encuentra regido por los principios de universalidad y gratuidad, y al interior de los cuales sólo la lógica sanitaria determina la aceptación o no de un paciente a la institución. Es decir, un principio jerárquico es el que garantiza el correcto funcionamiento de la red.

Por el contrario, el principio de la autogestión introduce al sistema una lógica de competencia en detrimento de la citada lógica jerárquica. Ya no sólo es la lógica sanitaria la que determina la pertinencia o no de la atención de un determinado paciente en una institución, sino también su capacidad de pago. Además, la institución deja de tener un rol "pasivo" en la red de efectores sanitarios a la espera de los pacientes que "le corresponden" en función de su lugar en el escalonamiento de referencia y contrarreferencia, y asume un rol "activo" en la búsqueda de todos aquellos potenciales pacientes que, independientemente del nivel de complejidad que requieran, disponen de alguna capacidad de pago.

Es notable observar cómo esta confrontación entre lógica jerárquica y lógica de competencia dificulta la circulación de los pacientes a través de las "rutas" previamente establecidas. En definitiva, la mayoría de los pacientes, en particular los carenciados, más allá de las complejidades hospitalarias, terminan siguiendo "la ruta" de la gratuidad, en tanto criterio de elección de una institución sanitaria.

\subsection{LA "CLIENTELIZACIÓN" DE LA POLÍTICA SANITARIA}

Las políticas sociales en América Latina han estado generalmente puestas al servicio de la construcción de clientelas electorales. El favoritismo, el nepotismo, el amiguismo, el clientelismo y la arbitrariedad son todos rasgos comunes a la mayoría de los países latinoamericanos.

Sin embargo, aquellas políticas que adquirieron patrones de mayor universalidad en la provisión de los servicios habían logrado minimizar este rasgo propio de las políticas de la región. Ejemplo de ello han sido, históricamente, las políticas de salud y educación.

En este sentido, la descentralización, en el caso de la salud, se ha caracterizado por haber colocado a la política sanitaria en una relación de mucha mayor dependencia del poder político local. La discrecionalidad, el asistencialismo y la clientelización han pasado a ser elementos constitutivos centrales de las políticas de salud locales. Estos aspectos habían estado, al menos, opacados en comparación con otras áreas de la política social, cuando la política sanitaria dependía de los niveles provinciales o nacionales. La descentralización los transparentó de una manera radical.

La dependencia directa del área sanitaria de la figura del intendente se evidencia al analizar las estructuras organizacionales de los municipios o comunas, en particular las de menor tamaño. En general, el efector de salud tiene una dependencia política y funcional directa del intendente sin ningún tipo de intermediación. El director o encargado del efector de salud se reporta de manera directa al mismo sin necesidad de seguir los niveles jerárquicos previos.

Existe, además, un consenso general a nivel local acerca de que la política de salud se ha transformado en un eslabón central de cualquier estrategia de legitimación política. Hoy, el impacto político de lo que sucede o deja de suceder en un efector sanitario se ha hecho mucho mayor en comparación a cuando estos efectores pertenecían a la jurisdicción provincial. Los servicios que se prestan en un centro de salud, y la forma en que se lo hace, pueden determinar los resultados de una elección local. 
La provisión de servicios de salud, junto con los que se ofrecen en otras áreas de la política social, pasa a conformar el complejo entramado de intercambios directos que se realizan entre "la intendencia" y "la comunidad", y que son útiles a la construcción de lealtades y clientelas que permiten la reproducción de los grupos de poder locales.

\subsection{ESTRATEGIAS DE FINANCIAMIENTO}

La descentralización de servicios, se suponía, debía provocar una mejor asignación de los recursos destinados al sector público provincial de la salud. Sin embargo, con relación a este punto, a nivel local encontramos una seria dificultad para precisar "cuánto" y "cómo" se gasta debido a las múltiples fuentes de sus recursos (nacionales, provinciales, municipales y comunitarios) y a la imprecisión de sus sistemas de información.

Lo que sí es posible determinar es que con la descentralización ha disminuido sustancialmente el compromiso presupuestario de la jurisdicción provincial con los efectores descentralizados, por lo cual son los municipios y la propia sociedad quienes deben compensar con recursos propios la menor disponibilidad de recursos provinciales.

Así, la mayoría de municipios y comunas han instrumentado mecanismos de financiamiento directo de los servicios por parte de la comunidad. De esta forma, el sistema local ha pasado en pocos años de ser financiado predominantemente a través de subsidios a la oferta y casi exclusivamente con recursos presupuestarios, a ser financiado a través de una multiplicidad de estrategias en donde los recursos presupuestarios puros son sólo una porción.

Las maneras de instrumentar el financiamiento "alternativo" o el aporte comunitario hacia el servicio de salud son variadas:

- Conformación de cooperadoras que recaudan fondos comunitarios y establecen aranceles mínimos a las prestaciones de salud.

- Conformación de fundaciones que canalizan aportes y donaciones de los sectores de altos ingresos de la comunidad.
- Hospital de autogestión que permite la facturación al Sistema de Seguridad Social Nacional.

- Pago directo de consultas médicas ("especialidades") con honorarios diferenciales según el nivel socio económico del paciente (una suerte de "Medicina Privada" para pobres).

- Sistemas de cobros intermunicipales utilizados para financiar las prestaciones de los carenciados de localidades vecinas.

- Tercerización de estudios complementarios (laboratorio y estudios por imágenes, principalmente) que son abonados con tarifas diferenciales según capacidad de pago. El municipio sólo abona la tarifa de los carenciados.

- Creación de tasas municipales específicas para financiar el establecimiento sanitario de la localidad

En realidad, se puede decir que esta multiplicidad de sistemas alternativos de financiación de los establecimientos sanitarios no sólo, sirven para desplazar parte de ellos a la propia comunidad, lo que no es poco importante, sino también para lograr otros objetivos.

Pues bien, estos sistemas han logrado instalar en la mayoría de los actores involucrados e incluso en la comunidad, una fuerte "cultura de gasto" en relación con los servicios de salud, cultura inexistente cuando los efectores pertenecían a la jurisdicción provincial. La idea predominante es que el municipio puede instrumentar ampliaciones de servicios pero no "hacerse cargo" de la totalidad de su sostenimiento.

Existe, además, una necesidad creciente de transparentar los subsidios a los carenciados, los únicos que se considera con derecho a la gratuidad. Es común la determinación de aranceles diferenciales u honorarios que el municipio paga "por" los pobres. Los carenciados concurren a consultas en donde los profesionales le facturan al municipio por los pobres atendidos. Estos sistemas están sirviendo para instrumentar modalidades de subsidio a la demanda en donde "el dinero sigue al paciente". Hemos hablado ya de las dificultades que esto genera a la hora de construir redes de efectores de complejidad creciente en donde la lógica sanitaria sea la que establezca la "división del trabajo". 


\subsection{GESTIÓN DEL RECURSO HUMANO}

La problemática de la gestión del recurso humano ha sido sumamente compleja para la mayoría de los municipios que tomaron a su cargo establecimientos sanitarios. Esto ha sido así porque los gobiernos locales se vieron ante el imperativo de tener que absorber planteles completos de personal sanitario, manteniendo las condiciones de contratación preexistentes sin tener, al menos, alguna experiencia al respecto.

Así, la provincia de Córdoba había sancionado en el año 1988 la Ley $N^{\circ} 7625$ del Régimen del Personal que integra el Equipo de Salud Humana. Esta ley establecía las condiciones disciplinarias de ingreso, ascenso y permanencia del personal sanitario de la provincia, además de estructurar un nuevo sistema escalafonario para el mismo. Esta ley se rige, esencialmente, por los principios de mérito para el ingreso, capacitación permanente, carrera sanitaria y estabilidad en el cargo. El personal transferido se encontraba actuando en el marco de este régimen laboral. En este sentido, las modalidades de permanencia en el sistema se limitaban a las condiciones de personal de planta permanente o no permanente (incluye contratado, interino, y suplente).

En este marco, la provincia transfiere a los municipios sólo personal permanente porque, a los fines de disminuir el número de personal que debían absorber aquellos al momento del traspaso, la jurisdicción provincial cesa a todos los trabajadores no permanentes. Los municipios se comprometen a mantener las condiciones laborales del personal absorbido.

A partir de este momento, los municipios inician un lento pero constante proceso de transformación de las condiciones de contratación del personal de los establecimientos sanitarios. Los cargos del personal permanente que se liberan por jubilaciones o renuncias no son reemplazados en iguales condiciones.

El propósito de este proceso ha sido flexibilizar las condiciones de contratación o modalidades de incorporación de personal a los establecimientos con el fin de disminuir la carga presupuestaria destinada al personal y evitar la situación de "relación de dependencia" con el municipio, lo que disminuye el riesgo laboral para el mismo.
En este sentido, es posible encontrar al personal que cumple tareas para el municipio en el área de Salud en las siguientes condiciones:

- Personal de Planta Permanente.

- Personal Contratado.

- Personal con contrato de Locación de Servicio.

- Becarios de Programas Nacionales (Médico de Cabecera, Médicos Promotores, Médicos Comunitarios).

- Convenios de trabajo por los cuales el personal utiliza la infraestructura hospitalaria pero cobra por sus prestaciones honorarios diferenciales según la capacidad de pago del paciente. El municipio sólo les paga lo correspondiente a las prestaciones dirigidas a los carenciados.

- Convenios con profesionales que trabajan en sus consultorios privados pero atienden en los mismos un cupo diario de carenciados por los cuales le facturan al municipio.

- Convenios de tercerización de servicios, en particular prácticas complementarias. El municipio abona al privado por las prácticas realizadas a carenciados.

- Convenios de comodato entre los profesionales de salud y el municipio. Estos convenios implican que los profesionales asumen la responsabilidad del funcionamiento del Centro de Salud y a cambio reciben un pago mediante la utilización de una fórmula polinómica que tiene en consideración cantidad y tipo de prestaciones. Esta fórmula incluye mínimos y topes en cuanto a la cantidad exigida de prestaciones.

- Beneficiarios del Plan Jefes y Jefas de Hogar que fueron capacitados como agentes sanitarios.

La transformación de las modalidades de contratación, además de lo ya expresado, le permite a los municipios ir abandonando la "relación de dependencia" y el asalariamiento de su personal de salud, en particular el de los médicos. De esta forma, los gobiernos locales avanzan en modos de pagos por trabajo. Esto le permitiría mejorar la productividad del trabajo profesional y evitar la políticamente costosa tarea de controlar el trabajo profesional ya que, a nivel local, se considera que el asalariamiento médico se había transformado en un desincentivo al trabajo y generador de baja productividad del mismo. Los problemas de no cumplimiento de las cargas 
horarias o la desviación de pacientes desde el sector público al privado aparecen como los problemas más citados para explicar la disconformidad municipal con las modalidades de contratación heredadas de la administración provincial.

\subsection{PARTICIPACIÓN SOCIAL}

La descentralización de los servicios de salud tenía como uno de sus objetivos centrales la democratización del sector a partir de la apertura del mismo a la participación comunitaria. Se entendía la participación no sólo en el sentido de colaborar en la implementación de las políticas sanitarias, sino que también debía abrirse a la planificación, diseño y evaluación de las mismas.

En la provincia de Córdoba los resultados al respecto han sido francamente decepcionantes. Así, en los municipios no se observan canales de participación formales o informales que signifiquen la existencia de un proceso de involucramiento de la ciudadanía en la cuestión de la salud.

Por el contrario, la transferencia de los servicios de salud a nivel local, en lugar de movilizar un gradual pero sostenido proceso de redistribución del poder social, ha sido útil a los fines de consolidar el poder político de personas o grupos de la región.

Si la propuesta teórica de la descentralización pensaba que la política de salud podría ser un espacio para los que no tenían poder, finalmente la misma terminó siendo una poderosa herramienta al servicio de los que ya lo tenían.

Las únicas experiencias que aparecen, a los ojos de algunos entrevistados como participativas son las cooperadoras hospitalarias. Sin embargo, al analizar el funcionamiento de las mismas se comprueba que tampoco son espacios abiertos y democráticos: por el contrario, suelen convertirse en lugares de poder siempre ocupados por las mismas personas de la comunidad. Pero aún más: en muchos casos, al interior de estas organizaciones se terminan reproduciendo prácticas de ejercicio del poder que, de ser observadas en otros ámbitos, generarían el mas amplio repudio.
En algunos casos, también las cooperadoras han funcionado como un verdadero "contrapoder" para el intendente, quien visualiza a dichas instituciones como un obstáculo para su gestión y por lo cual se las termina desactivando. En general, este es el caso de aquellos municipios en los que en la actualidad no hay cooperadoras colaborando con el efector sanitario.

En síntesis: si la descentralización debía estar al servicio de la democracia y la participación, en los hechos terminó siendo una política al servicio de la consolidación del statu quo local.

\section{ALGUNAS CONCLUSIONES}

En primer lugar, es posible concluir que, luego de lo ya dicho en el trabajo, el proceso de descentralización de servicios de atención médica ha sido la política de reforma sectorial más importante de las últimas décadas en la provincia de Córdoba. A partir de ella se reconfigura la estructura pública de salud, pasando a ocupar la jurisdicción provincial un lugar menos relevante en su rol de prestador.

También, la política descentralizadora ha sido sostenida desde hace más de 15 años por gobiernos de distinto signo político. Ha sido la única política de reforma sectorial que ha gozado de un alto nivel de consenso entre los actores políticos provinciales.

Sin embargo, lo que ha ido cambiando a lo largo de los años fueron las condiciones políticas que le posibilitaron a los diferentes gobiernos impulsar la política de descentralización con distintas modalidades y énfasis.

Aunque el proceso se inicia en el contexto de las propuestas de modernización del estado provincial, y la descentralización se presenta como una política al servicio de la democratización de la gestión pública, el impulso final de la misma se produce debido a las urgencias financieras y fiscales de la provincia en 1995. La lógica del ajuste terminó impregnando a la mayor parte del proceso, lo cual, como decimos en el título del artículo, ha provocado una enorme frustración con relación a las expectativas generadas inicialmente.

Esto queda evidenciado en el hecho de que el resultado más tangible que se ha obtenido 
al final de la municipalización de efectores sanitarios ha sido la notable reducción del compromiso presupuestario provincial con el sector, pasando desde el $17 \%$ del total de los recursos presupuestarios provinciales en la década del '80 (14) hasta el $9 \%$ en la actualidad. La contracara de este proceso ha sido el logro de un mayor involucramiento por parte de los municipios y comunidades locales en el financiamiento de sus servicios de salud.

Ahora bien, el hecho de que haya sido la lógica "economicista" la que predominó a lo largo de la mayor parte del proceso debe ser analizado en toda su complejidad. Además debe considerarse que el proceso de municipalización de efectores se aceleró a partir de 1995 en el marco de un estilo de gestión personalista y autoritario que sacó al proceso de descentralización de una suerte de "estado de estancamiento".

En este sentido, debe decirse que lo sucedido en Córdoba a partir de 1995 estaría en sintonía con una parte importante de la bibliografía nacional e internacional sobre reformas estatales, que considera que las crisis fiscales y financieras profundas son una "ventana de oportunidad" para llevar adelante transformaciones radicales (reformas de shock) de las instituciones públicas y que los estilos de gestión decisionistas serían los que están en mejores condiciones de aprovechar estos escenarios propicios para el cambio. La profundidad de la crisis acotaría los márgenes de resistencia de los actores potencialmente perjudicados por las reformas y permitiría avanzar en la dirección deseada con el consenso plebiscitario de la ciudadanía, que sólo aspira a una rápida restauración del orden social y político (15).

Dicho lo anterior, se podría afirmar que si el objetivo del equipo reformista durante el que hemos denominado segundo período de la descentralización provincial, era el de concluir con la transferencia de efectores sanitarios de baja complejidad hacia los municipios, habrá que admitir que el resultado fue exitoso. Al inicio de 1996, la jurisdicción provincial ya no tenía ninguna institución de salud "descentralizable". Es decir, el proceso de transferencias a los municipios y comunas estaba concluido.

Sin embargo, si la descentralización fue pensada como una estrategia al servicio de una verdadera transformación del sistema de salud provincial, allí los resultados han sido más que decepcionantes.

La urgencia fiscal y la necesidad de "descargar" de forma perentoria el financiamiento de una gran cantidad de servicios de salud sobre otros actores impidió que el nivel central pudiera acompañar el proceso, desarrollando estrategias de coordinación, normatización y supervisión que hubieran permitido lograr mejores resultados sanitarios. Por el contrario, la modalidad compulsiva de descentralización generó un alto nivel de conflicto entre la provincia y los municipios, lo que no permitió implementar acciones de cooperación. Es recién a partir del año 1999, con el cambio de gobierno, que la jurisdicción provincial, con mucha dificultades, empieza a reconducir este proceso.

La descentralización ha profundizado algunos de los peores rasgos del sistema anterior. La falta de coordinación, la desintegración, la atomización, la fragmentación, la irracionalidad en el uso de los recursos, los problemas de financiamiento y el fortalecimiento del modelo médico tradicional son algunos de los resultados más significativos. A esto deben agregarse nuevos problemas, como la "clientelización" de la política de salud, la falta de información sanitaria en el nivel central y el cierre arbitrario de algunos servicios.

Además, la falta de una instancia superior que coordinase y supervisara el proceso dejó librado a cada municipio a que construyera "sus" propias respuestas sanitarias frente a la problemática que enfrentaban. Así se profundizó la heterogeneidad en la fragmentación, lo que dificulta aún más las posibilidades de coordinación desde el nivel central.

En este contexto de pobres resultados, se hace difícil pensar que la descentralización haya sido un instrumento al servicio de una mayor eficiencia, eficacia, calidad y participación en el campo de la salud, mas allá de que los municipios y comunas, en una importante cantidad, han logrado sostener y ampliar sus servicios de salud con enorme esfuerzo.

Ahora bien, en este punto no debe dejar de mencionarse el tema del desarrollo de capacidades estatales a nivel local. En este sentido, si entendemos por capacidad estatal la aptitud de las instancias de gobierno para plasmar, a través de 
las políticas públicas, los máximos niveles posibles de valor social, es posible afirmar entonces que la misma puede ser "visibilizada" a través de una serie de atributos que caracterizan a las respuestas sobre las cuestiones prioritarias. Así, la capacidad estatal se expresa mediante atributos tales como, coordinación, flexibilidad, innovación, calidad, sostenibilidad, evaluabilidad, eficiencia, eficacia y rendición de cuentas (accountability) (16).

A lo largo de nuestro trabajo ha quedado planteado que durante el proceso de descentralización ha sido poco lo realizado por la jurisdicción provincial en el sentido de favorecer el desarrollo de capacidades estatales (políticas y administrativas) que permitiesen mejorar los resultados de la política descentralizadora.

Sin embargo, ha quedado dicho también que a pesar de la "ausencia" del estado provincial, los municipios han debido desarrollar capacidades estatales administrativas para adaptarse a las nuevas condiciones y restricciones imperantes a partir de la descentralización. En ese sentido, ha sido rico lo desarrollado en términos de flexibilidad, innovación y sostenibilidad, no así lo logrado en términos de coordina- ción, evaluabilidad, calidad, eficiencia, eficacia y rendición de cuentas.

Finalmente, debemos decir que así como consideramos que la modalidad que adquirió el proceso descentralizador fue responsable de la profundización de la fragmentación sectorial y del incremento de la heterogeneidad al interior de la misma, también debemos concluir que este factor no alcanza para explicar las particularidades que asumieron las instituciones sanitarias a nivel local luego de la descentralización.

Es decir, sometidos a la misma lógica descentralizadora, cada municipio fue construyendo su propia institucionalidad sanitaria en función de las estrategias desarrolladas por cada uno de los actores locales. Esta lógica implica el conflicto y la confrontación de ideas, proyectos e intereses que da por resultado una nueva institucionalidad.

El presente trabajo deja planteada la necesidad de avanzar en futuras investigaciones que permitan construir los marcos analíticos que nos faciliten una mejor y más acabada comprensión de los procesos de construcción institucional a nivel local.

\section{NOTAS FINALES}

a. El presente trabajo está basado en el informe final de la investigación "La descentralización de servicios de salud en Córdoba. ¿Lógica sanitaria o lógica económica?" realizada con el apoyo de la Beca Ramón Carrillo - Arturo Oñativia 2004, otorgada por el Ministerio de Salud y Ambiente de la Nación. Agradezco los valiosos comentarios realizados por Susana Belmartino durante la elaboración del mismo.

b. La provincia de Córdoba es una de las 23 provincias que conforman la República Argentina, tiene una ubicación mediterránea y céntrica y una extensión de $165.321 \mathrm{Km}^{2}$, lo que la constituye en la quinta provincia por su dimensión. Según los datos del Censo Nacional de 2001, tiene una población de 3.066.801 habitantes, lo que da una densidad poblacional de 18,6 habitantes por $\mathrm{Km}^{2}$. El mismo Censo informa que en dicho año el $13,5 \%$ de sus habitantes viven en hogares con Necesidades Básicas Insatisfechas.
Según datos del Instituto Nacional de Estadísticas y Censos, Córdoba tiene un 12,3\% de desocupados y un $8,1 \%$ de subocupados. El sistema de salud provincial reproduce las características nacionales, al estar dividido en tres sub-sectores: estatal, seguridad social y privado. La provincia tiene, según datos del Censo 2001, el 45,76\% de su población sin cobertura de seguro de salud, encontrándose casi tres puntos por debajo del promedio nacional $(48,05 \%)$ en dicho momento. Como referencia de la situación sanitaria provincial vale decir que la Tasa de Mortalidad Infantil para el año 2004 se ubicó en el 12,7 por mil nacidos vivos, casi dos puntos porcentuales por debajo del promedio nacional $(14,4$ por mil nacidos vivos). A pesar de las recurrentes crisis económicas y sociales que han vivido el país y la provincia en los últimos veinte años, esta tasa ha venido disminuyendo de manera sistemática, ya que en el año 1987 estaba en el 25,1 por mil nacidos vivos. 


\section{REFERENCIAS BIBLIOGRÁFICAS}

1. Ase I, Burijovich J. La Reforma del Sector Salud en Córdoba. El naufragio de un piloto de tormentas. Revista Administración Pública y Sociedad. 2000;13:19-39.

2. Burijovich J, Pautassi L. Equidad de género y calidad en el empleo: las trabajadoras y los trabajadores en salud en Argentina. Chile: CEPAL; 2001. (Serie Mujer y Desarrollo; No 30).

3. Ugalde A, Homedes N. Descentralización del sector salud en América Latina. Cuadernos Médico Sociales. 2002;81:15-30.

4. Reppetto F. Descentralización de la Salud Pública en los noventa: una reforma a mitad de camino. Argentina: Centro de Estudios para el Desarrollo Institucional. Fundación Gobierno y Sociedad, Fundación Grupo Sophia; 2001. Documento 55.

5. Organización Panamericana de la Salud. Desarrollo y Fortalecimiento de los Sistemas Locales de Salud. Temas de Discusión. Washington: OPS; 1993. (Serie HSS/SILOS vol17).

6. Filmus D. Estado, Sociedad y Educación en la Argentina de fin de siglo. Procesos y desafíos. Buenos Aires: Editorial Troquel; 1996.

7. Gagliano E, Rosenfeld A, Tillea T. Fundamentos de la propuesta de descentralización y desconcentración de la atención estatal en salud en Córdoba. Ponencia presentada en el VIII Congreso Nacional de Administración Pública; 27,28 de Noviembre de 1988; Córdoba, Argentina. Córdoba: IIFAP; 1989.

8. Ahumada J, Miretti L. Seguimiento de Servicios de Salud Descentralizados. Córdoba: Gobierno de la Provincia de Córdoba; 1993.
9. Subsecretaría de la Función Pública de la Provincia de Córdoba. Cuadernos de la Reforma del Estado. Córdoba en Cifras. Córdoba: Gobierno de la Provincia de Córdoba; 1994. (Serie Información).

10. Angeloz E. La Reforma Democrática en el Estado Provincial. Córdoba: Difusión AB; 1993.

11. Cingolani M. Las Relaciones intergubernamentales Central-locales e Inter-locales y la Gestión de Servicios Descentralizados. Ponencia presentada en el VI Congreso Internacional del CLAD; 5 al 9 de Noviembre de 2001; Buenos Aires, Argentina. (Mimeo).

12. Instituto Nacional de Estadística y Censos. Censo Nacional de Población 2001. Argentina: INDEC; 2001.

13. Belmartino S, Bloch C, Báscolo E. Reforma de la atención médica en escenarios locales (1990 2000). Buenos Aires: Lugar Editorial; 2003.

14. Navarro M, Rosenfeld A. El sector salud de la Provincia de Córdoba. Buenos Aires: Fondo de Cultura Económica; 1993.

15. Corrales J. ¿Contribuyen las crisis económicas a la implementación de reformas de mercado? La Argentina y Venezuela en los '90. Revista Desarrollo Económico.1999;153(39):3-29.

16. Repetto F. Capacidad estatal: requisito necesario para una mejor política social en América Latina. Ponencia presentada en el VIII Congreso Internacional del CLAD sobre la Reforma del Estado y de la Administración Pública; 28 al 31 de Octubre de 2003; Ciudad de Panamá, Panamá. (Mimeo).

\section{BIBLIOGRAFÍA DE CONSULTA}

Acuña C, Chudnovsky M. Análisis de la dinámica político-institucional y organizacional del Área Materno Infantil (con énfasis en el Programa Materno Infantil y Nutrición - PROMIN). Argentina: Centro de Estudios para el Desarrollo Institucional, Fundación Gobierno y Sociedad; 2002.
Belmartino S. Nuevas reglas de juego para la atención médica en la Argentina. ¿Quién será el árbitro? Buenos Aires: Lugar Editorial; 1999.

Cetrángolo O, Devoto F. Reformas en la política de salud en la Argentina durante los años noventa con especial referencia a la equidad. Buenos Aires: Centro de Estudios para el Cambio Estructural; 1998. (Serie de Estudios CECE; N²7). 
Cunill Grau, N. Repensando lo Público a través de la Sociedad. Nuevas formas de gestión pública y representación social. Venezuela: CLADNueva Sociedad; 1997.

Di Gropello E, Cominetti R. La descentralización de la educación y la salud. Un análisis comparativo de la experiencia latinoamericana. Santiago de Chile: CEPAL; 1998.

Finot I. Descentralización en América latina: ¿Cómo conciliar eficiencia con la equidad?. Revista del CLAD Reforma y Democracia. 1998;10:129-148.

Franco R. Descentralización, Participación y Competencia en la Gestión Social. Ponencia presentada en el VIII Congreso Internacional del CLAD sobre la Reforma del Estado y de la Administración Pública; 28 al 31 de Octubre de 2003; Ciudad de Panamá, Panamá. (Mimeo).

García Delgado D. Estado y Sociedad. La nueva relación a partir del cambio estructural. Argentina: Grupo Editorial Norma; 1994.
La Serna C. Actores y procesos en la crisis del Estado de Bienestar. Revista Administración Pública y Sociedad. 1998;10: 101-121.

Novick M, Galin P. Flexibilidad del mercado de trabajo y precarización del empleo. El caso del sector salud. Observatorio de Recursos Humanos en Salud. 2003;58:11- 90.

Organización Mundial de la Salud. Declaración de Alma-Ata. Procedente de la Conferencia Internacional sobre Atención Primaria de la Salud [Documento Final]; 6 al 12 de Septiembre de 1978; Alma-Ata, URSS. Ginebra: OMS; 1978.

Sojo A. Reformas de gestión en salud en América Latina: los cuasi-mercados de Colombia, Argentina, Chile y Costa Rica. Chile: CEPAL; 2000. (Serie Políticas Sociales; No39)

Vasallo C. El estado de las reformas en las provincias. Ponencia presentada en el Encuentro Internacional sobre Seguro Público de Salud; 7, 8 de Mayo de 1998; Bariloche, Argentina. Buenos Aires: Ediciones ISALUD; 1998.

\section{FORMA DE CITAR}

Ase I. La Descentralización de Servicios de Salud en Córdoba (Argentina): Entre la Confianza Democrática y el Desencanto Neoliberal. Salud Colectiva. 2006;2(2):199-218.

Recibido el 19 de septiembre de 2005

Versión final presentada el 01 de diciembre de 2005

Aprobado el 2 de febrero de 2006 